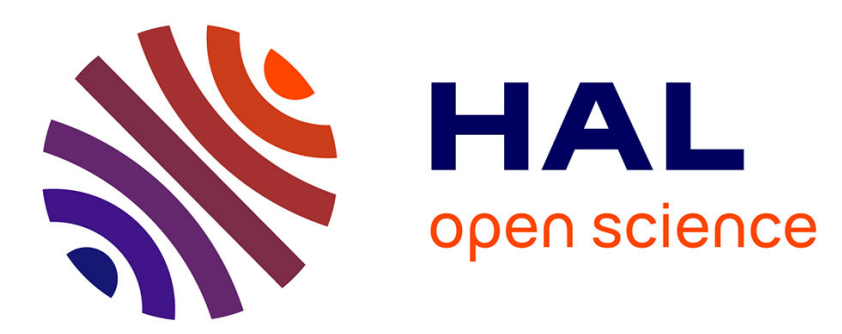

\title{
Normal adhesive contact on rough surfaces: efficient algorithm for FFT-based BEM resolution
}

\author{
Valentine Rey, Guillaume Anciaux, Jean-François Molinari
}

\section{To cite this version:}

Valentine Rey, Guillaume Anciaux, Jean-François Molinari. Normal adhesive contact on rough surfaces: efficient algorithm for FFT-based BEM resolution. Computational Mechanics, 2017, 60 (1), pp.69-81. 10.1007/s00466-017-1392-5 . hal-01755724

\section{HAL Id: hal-01755724 \\ https://hal.science/hal-01755724}

Submitted on 30 Mar 2018

HAL is a multi-disciplinary open access archive for the deposit and dissemination of scientific research documents, whether they are published or not. The documents may come from teaching and research institutions in France or abroad, or from public or private research centers.
L'archive ouverte pluridisciplinaire HAL, est destinée au dépôt et à la diffusion de documents scientifiques de niveau recherche, publiés ou non, émanant des établissements d'enseignement et de recherche français ou étrangers, des laboratoires publics ou privés. 


\title{
Normal adhesive contact on rough surfaces: efficient algorithm for FFT-based BEM resolution
}

\author{
Valentine Rey ${ }^{1} \quad$ Guillaume Anciaux ${ }^{1}$ \\ Jean-François Molinari ${ }^{1}$ \\ ${ }^{1}$ Laboratoire de simulation en mécanique des solides, Institut ENAC \\ Ecole Polytechnique Fédérale de Lausanne (EPFL) \\ Station Z \\ CH-1015 Lausanne
}

\begin{abstract}
We introduce a numerical methodology to compute the solution of an adhesive normal contact problem on rough surfaces with the Boundary Element Method. Based on the Fast Fourier Transform and the Westergaard's fundamental solution, the proposed algorithm enables to solve efficiently the constrained minimization problem: the numerical solution strictly verifies contact orthogonality and the algorithm takes advantage of the constraints to speed up the minimization. Comparisons with the analytical solution of the Hertz case prove the quality of the numerical computation. The method is also used to compute normal adhesive contact between rough surfaces made of multiple asperities. keywords:Adhesive normal contact, BEM, rough surfaces
\end{abstract}

\section{Introduction}

Natural surfaces are rough and composed of many asperities over a wide range of length scales. Contact mechanics of rough surfaces need to account for the complex interactions between these asperities. Numerical simulations can reveal how the true contact area (which is a small fraction of the nominal contact area), the distribution of contact clusters sizes, and the distribution of local pressures, amongst other quantities of interest, vary with load and surface roughness $[31,21,23,3,22,14,4,26,6]$. A fundamental understanding of the micro contacts finds many applications including friction, wear, or the thermal or electrical conductivity of an interface. At the small scale, an additional complexity is brought 
by non-negligible Van der Waals forces. Adhesion is of primary concern for the robustness and reliability of micro-electromechanical systems (MEMS) and nanoelectromechanical systems (NEMS). It needs to be accurately accounted for in numerical methods.

Classical theories describing adhesion for the case of Hertz contact have been developed several decades ago $[17,15,10]$. However, these models only consider a single asperity and therefore do not account for elastic interactions between asperities, although it is known $([12,11,4,20])$ that neglecting these interactions leads to wrong estimates of the true contact area. Several numerical approaches including adhesion for contact between rough surfaces have been developed in the past decades. These include the diffusion model of Persson [24], the BoundaryElement Method (BEM) [5, 6, 7], and the Green's Function Molecular Dynamics method (GFMD) $[18,21]$. Resolving accurately and efficiently for the true contact area in the presence of adhesive forces yield several numerical challenges. Yet, to the best of the authors' knowledge, the current literature does not explain in detail the coupling of adhesive forces with inequality contact constraints for the BEM.

The objective of this paper is therefore to fill this void. We present an efficient algorithm to compute the solution of the frictionless normal adhesive contact problem within the Boundary Element Method (BEM). Because of the use of BEM, we will only consider elastic linear behaviour. Including material non-linearities such as plasticity is a an additional difficulty that can be addressed with Finite Element Method for example. Interested readers may refer to [30]. Here, we present an algorithm that is composed of two nested conjugate gradients. It enables to solve adhesive normal contact by ensuring strictly the contact inequality and behaves better than penalization techniques, which relax the contact inequality by authorizing small interpenetration [30].

In section 2 , we describe the mechanical model. Then, in section 3 , the problem is discretized and the algorithms employed to solve the constrained minimization problem are presented. The first part of section 4 is dedicated to the validation of the proposed method by comparing our numerical results to analytical solutions in the Hertzian case. The second part of section 4 consists in the comparison of contact maps for rough surfaces with and without adhesive behaviour. Section 5 concludes the paper.

\section{Mechanical model}

We study the frictionless normal contact between two homogeneous semi-infinite solids: one flat elastic isotropic deformable solid (under small displacements hypothesis) of Young's modulus $E$ and Poisson's ratio $\nu$ and a rigid (infinitely stiff) rough surface with an height profile $h$. 


\subsection{Continuous formulation}

We use an integral formulation [26] that enables to compute the solution in the whole domain from the normal displacement field $u$ obtained at the surface $S$ of the flat deformable solid. The problem is therefore solved only at the surface, which represents a reduction of dimension with the obvious advantage to reduce the computational cost. Integral representations can be used to compute quantities inside the bulk (displacements in the bulk for instance) as a post-processing step. The elastic behaviour is described through a convolution product with a kernel defined from a fundamental solution.

More precisely, the link between normal displacement $u(\underline{x})$ and the normal pressure $p(\underline{x})$ at the surface is:

$$
u(\underline{x})=\int_{S} K\left(\underline{x}-\underline{x}^{\prime}\right) p(\underline{x}) d \underline{x}^{\prime}
$$

If we use the Boussinesq's fundamental solution, $K(\underline{x})$ is the surface deflection at the point of coordinate $\underline{x}$ produced by a concentrated normal load of unit magnitude, applied at the surface at the origin $(0,0)$. In this paper, we decide to use the Westergaard's solution [29] that connects pressure to displacements in Fourier's space thanks to influence coefficients.

We note $g$ the gap defined by:

$$
g(\underline{x})=u(\underline{x})-h(\underline{x})
$$

The condition of non interpenetration between the two solids imposes that

$$
\forall \underline{x}, g(\underline{x}) \geqslant 0
$$

The prescribed loading is given in terms of the mean value of the gap:

$$
\frac{1}{S} \int_{S}(u(\underline{x})-h(\underline{x})) d S=g_{0}
$$

In the following, we first give the formulation of the contact problem without adhesion before introducing adhesive interactions in section 2.3.

\subsection{In absence of adhesion}

The displacement field $u$ is the solution of the minimization problem:

$$
u=\underset{u \in \mathcal{A}}{\operatorname{argmin}} E_{p}(u)
$$


where $\mathcal{A}$ is the space of admissible displacement fields characterized by:

$$
u \in \mathcal{A} \Leftrightarrow \forall x, u(\underline{x})-h(\underline{x}) \geqslant 0 \text { and } \frac{1}{S} \int_{S}(u(\underline{x})-h(\underline{x})) d S=g_{0}
$$

When there is no adhesion, the potential energy is equal to the elastic energy:

$$
E_{p}=E_{\text {elas }}=\frac{1}{2} \int_{S} u(\underline{x}) p(\underline{x}) d S
$$

In the space $\mathcal{A}$, the inequality constraint corresponds to inter-penetration restrictions and the equality condition corresponds to the applied load. The wellknown Hertz-Signorini-Moreau orthogonality [27] for contact reads:

$$
\forall \underline{x}, \quad p(\underline{x}) g(\underline{x})=0, \quad p(\underline{x}) \geqslant 0, \quad g(\underline{x}) \geqslant 0
$$

The pressure being always positive can be either interpreted physically (only compressive forces can exist since there is no adhesion) or mathematically (the pressure is actually the Lagrange multiplier associated to the constraint of non-inter penetration). Let us note that this is a condition that connects the primal variable (the gap $g$ ) to the dual variable (the pressure $p$ ).

\subsection{In presence of adhesion}

When adhesion is considered, the potential energy is the sum of elastic energy and adhesive energy:

$$
E_{p}=E_{\text {elas }}+E_{\text {adh }}
$$

where the adhesive energy is usually a function of the distance between the two surfaces:

$$
E_{a d h}=f(g)
$$

The displacement field $u$ is still the solution of the minimization problem

$$
u=\underset{u \in \mathcal{A}}{\operatorname{argmin}} E_{p}(u)
$$

Let us rewrite the constrained minimization by defining the Lagrangian:

$$
\mathcal{L}_{\lambda}(u)=E_{p}(u)-\int_{S} \lambda(u-h) d S
$$

where $\lambda$ is the Lagrange multiplier that has to be positive $\lambda \geqslant 0$. The stationarity of the Lagrangian reads:

$$
\frac{\partial \mathcal{L}_{\lambda}(u)}{\partial u}=p+\frac{\partial f}{\partial u}-\lambda=0
$$


As a consequence, the Lagrange multiplier is equal to the sum of the elastic pressure $\mathrm{p}$ and the adhesive force.

The orthogonality for adhesive contact now reads:

$$
\forall \underline{x}, \quad\left\{\begin{array}{l}
\left(p(\underline{x})+\frac{\partial f}{\partial u}\right) g(\underline{x})=0 \\
p(\underline{x})+\frac{\partial f}{\partial u} \geqslant 0 \\
g(\underline{x}) \geqslant 0
\end{array}\right.
$$

The equality can be explained by the Lagrangian: when the constraint is not saturated $(g>0)$, the Lagrange multiplier is null $(\lambda=0)$ and when the constraint is saturated $(g=0)$, the Lagrange multiplier is strictly positive $\lambda>0$. From a mechanical viewpoint, at points where the two surfaces are not in contact $(g>0)$, the sum of forces is zero (elastic forces equilibrate adhesion forces). At points where the two surfaces are in contact $(g=0)$, the sum of forces has to be strictly positive.

\subsection{Stability analysis}

Adhesion may cause the loss of convexity of the potential energy, which could lead to unstable solutions [9]. Without adhesion, the second derivative of the potential energy is the elastic stiffness, which is a positive operator. As a consequence, the equilibrium solution is always stable. With adhesion, the second derivative of the energy may be negative. If it is the case, it implies that the equilibrium solution found by optimizing the energy is unstable. Many numerical techniques exist to compute bifurcated solutions and to study the stability of mechanical systems [19]. However, the contact constraint complicates this analysis since not all perturbations around an equilibrium position are admissible. This paper focuses on the computation of an equilibrium solution discarding the question of its stability. Nonetheless, we provide an outlook on the consequences of this issue on the algorithm we propose in subsection 3.3.

\section{Discretized problem and resolution algorithms}

Next, we present the discretized problem and algorithms to solve the adhesive contact.

\subsection{Discretized problem}

We consider a square surface of length L, which is discretized as a grid with the discretization length $l$. We aim at solving the continuous problem on the $N=\frac{L^{2}}{l^{2}}$ grid points (collocation) using BEM. We will denote by bold font $\mathbf{u}$ the vector 
collecting the $N$ nodal values of displacement and $\mathbf{p}$ the vector collecting the $N$ nodal values of pressure. The scalar $u_{i}$ is the value of the vertical displacement at the grid node $i$. We note $\mathbf{e}$ the vector whose each component is 1 .

The convolution product (1) is computed in Fourier's space so that the link between the displacement vector $\mathbf{u}$ and the elastic pressure vector $\mathbf{p}$ is the following:

$$
\mathbf{u}=\operatorname{FFT}^{-1}(\mathbf{K F F T}(\mathbf{p}))
$$

where FFT is the 2D Fast Fourier Transform, FFT $^{-1}$ is the Backward 2D Fast Fourier Transform, and $\mathbf{K}$ is a diagonal $N \mathrm{x} N$ matrix that contains the influence coefficients for the reference Westergaard solution [29]. These constant coefficients depend on the discrete set of frequencies admissible for the chosen discretization and on the Young's modulus $E$ (see [28] for their expressions). The use of Fast Fourier Transform (FFT) implicitly enforces periodic boundary conditions on the surface. In $\mathbf{K}$ the coefficient associated to the 0 -frequency is 0 . Indeed, the displacement of the surface is only known up to a rigid-body motion. We can also compute a centered pressure, that is to say a pressure with mean value 0 , from a displacement $\mathbf{u}$ using the relation:

$$
\mathbf{p}=\mathrm{FFT}^{-1}\left(\mathbf{K}^{-\mathbf{1}} \operatorname{FFT}(\mathbf{u})\right)
$$

We define the gap vector $\mathbf{g}=\mathbf{u}-\mathbf{h}$ and the admissible space

$$
\mathbf{u} \in \mathcal{A} \Leftrightarrow \forall i \in[1 ; N], g_{i} \geqslant 0 \text { and } \frac{1}{N} \sum_{i=0}^{N} g_{i}=g_{0}
$$

The discretized problem reads:

$$
\text { Find } \mathbf{u} \in \mathcal{A} \text { minimizing } E_{p}(\mathbf{u})
$$

\subsection{Resolution algorithm}

Solving the mechanical problem consists in minimizing a functional under equality and inequality constraints. Many strategies exist to solve this type of problem (see $[30,1]$ for a review of those techniques for contact problems). In our framework, since the rough surface is rigid, the projection of any trial solution onto the constrained space is straightforward. We will use a constrained conjugate gradient algorithm to solve the problem. As a consequence, when the solver has converged, the constraint is strictly verified (up to machine precision) and contact orthogonality is guaranteed. Instead of solving the problem with pressure as the unknown, we choose the gap to be the unknown. This choice is justified by the fact that the projection to satisfy the constraint on the gap is simple (the rough surface is 
rigid) and is independent of the adhesive potential. On the contrary, ensuring the constraint on the dual variable depends on the adhesive potential and may become much more difficult.

The algorithm we present in this paper is composed of two minimizations that are performed thanks to an iterative scheme. After initialization, the first search direction to minimize the potential energy is computed. The updated unknown vector may not verify the constraints and is therefore projected on the admissible space. Only when converged may the solution be the vector minimizing the potential energy and also respecting the constraints. To describe the algorithm, we first introduce $I$, the set of points in the grid, $I_{n a}$ the set of points $i$ where $g_{i}=0$ and $q_{i}<0$, where $\mathbf{q}$ is the vector defined as the sum of the elastic pressure $\mathbf{p}$ and the adhesive force:

$$
\mathbf{q}=\mathbf{p}+\frac{\partial f}{\partial \mathbf{u}}
$$

The subscript na stands for non admissible since the sum of elastic and adhesive pressures should always be positive (14). $I_{n c}$, where the subscript $n c$ stands for non contact, is the set of points $i$ where $g_{i}>0 . N_{n c}$ denotes the number of points in $I_{n c}$. $I_{c}$, where the subscript $c$ stands for contact, is the set of points $i$ where $g_{i}=0$. Obviously, $I_{n a} \subset I_{c}$. The contact zone is therefore described by $I_{c}$. At convergence, we should have $I_{n a}=\varnothing$ and $I_{n c}$ and $I_{c}$ forming a partition of $I$.

The algorithm (see Algorithm 1) is composed of two nested loops each corresponding to a minimization process. The objective of the outer loop is to minimize the potential energy $E_{p}$, which might create interpenetrated situations. The role of the inner loop is to project the unknowns on the admissible space (no inter penetration and enforcement of the targeted mean gap) and to minimize the orthogonality on the non contact set $I_{n c}$. Both minimizations are based on the classical conjugate gradient algorithm. The stopping criterion is defined using the scalar product $\epsilon=\sum_{i=1}^{N}\left|g_{i}\left(q_{i}-\frac{1}{N_{n c}} \sum_{I_{n c}} q_{i}\right)\right|=\sum_{i=1}^{N}\left|g_{i} q_{i}^{\prime}\right|$.

At the end of the algorithm, because equation (16) only gives a centered field, it is required to recover the mean value of the total pressure q. This is done at the end of the outer loop, by computing $p_{0}=-\min _{I} q_{i}$ so that the final total pressure is $\mathbf{q}+p_{0} \mathbf{e}$.

As a consequence, the fields computed verify contact inequalities. Indeed, $\forall i \in I_{c}, g_{i}=0$ and $\forall i \in I_{n c}, g_{i}>0$. Because of the definition of $p_{0}$, the final total pressure is such that $\forall i \in I,\left(q_{i}+p_{0}\right) \geqslant 0$.

Contact orthogonality is ensured thanks to the following property, whose conditions are verified at convergence.

Property 1. If $\forall i \in I_{c}, g_{i}=0, \forall i \in I_{n c}, g_{i}>0, I_{n a}=\varnothing$ and $\epsilon=0$ then $\forall i \in I_{n c},\left(q_{i}+p_{0}\right)=0$ with $p_{0}=-\min _{I} q_{i}$ 
Proof. First, since $\epsilon=0$, we know that $\forall i \in I_{n c}, q_{i}^{\prime}=0$. Therefore $\forall i \in I_{n c}$, $q_{i}^{\prime}=q_{i}-\frac{1}{N_{n c}} \sum_{I_{n c}} q_{i}=0$. As a consequence, on $I_{n c}, q_{i}$ is constant. Moreover, since $I_{n a}=\varnothing$, we have $\forall i \in I_{c}, q_{i}^{\prime} \geqslant 0$, so $\forall i \in I_{c}, q_{i} \geqslant \frac{1}{N_{n c}} \sum_{I_{n c}} q_{i}$. As a consequence, $\min _{I} q_{i}=\frac{1}{N_{n c}} \sum_{I_{n c}} q_{i}=q_{i, i \in I_{n c}}$.

Finally, on $I_{n c}$,

$$
\begin{aligned}
q_{i}+p_{0} & =q_{i}-\min _{I} q_{i} \\
& =\frac{1}{N_{n c}} \sum_{I_{n c}} q_{i}-\frac{1}{N_{n c}} \sum_{I_{n c}} q_{i} \\
& =0
\end{aligned}
$$

The fundamental following remarks explain how the algorithm can be rewritten in an elegant condensed form. First, the derivative of the energy functional and the derivative of $\mathcal{P}$ is $q$ in both cases. In the outer loop, $I_{n a}$ is empty so $I=I_{n c}+I_{c}$. As a consequence, the minimum of $E_{p}$ on $I_{n c}$ is the minimum of $E_{p}$ on $I$. Also, the two optimal scalars $\tau$ and $\alpha$ that are to be multiplied to the search direction in conjugate gradient iterations (see algorithms 1 and 2) are equal. Moreover, in the inner loop, $\mathcal{P}=0$ implies that the energy functional is minimum so that the test to stop the algorithm can be placed at the end of the inner loop. Finally, we can gather the loops by adding a new variable $\delta$ which is set to 1 when minimizing the energy functional, and to 0 when imposing the constraints. We thus obtain the condensed form of the algorithm (algorithm 3), which is similar to the pressure formulation presented in [25].

We highlight the fact that every search direction in the inner loop is a good search direction for the energy minimization. At convergence, the contact orthogonality is strictly verified. This would not have been the case with a penalization approach [30], where the non negativity of the gap is ensured weakly by penalizing negative gaps. A penalization approach requires the introduction of a scalar parameter representing an additional stiffness. The larger this stiffness, the lower the interpenetration. However, it is well-known that in order to obtain a negligible penetration, the penalty parameter has to be very large, which often leads to ill-conditioned problems since the ratio between the penalization parameter and the Young's modulus may become large. Uzawa algorithm [30] is based on an augmented Lagrangian formulation that enables to obtain no penetration with a finite penalization parameter. However, this algorithm requires to define the initial penalization parameter and the step size and is more complex to implement than the simpler formulation we present. Finally, since with those types of approach 
the orthogonality is not strictly enforced, the reconstruction for the total pressure from its centered counter-part may lead to wrong results.

The algorithm can only be used in the case of one deformable surface on a rigid surface. Indeed, if the two surfaces are deformable, many properties are lost. First, because the fundamental solution is no longer invariant by translation, the influence coefficients in matrix $\mathbf{K}$ depend on the point so that the computation of elastic behaviour becomes very expensive. Moreover, the detection of the contact zone is more difficult and the projection on the admissible space is not possible anymore. Alternative strategies have to be used (see [30] with active-set technique for example).

\subsection{Comments on stability issue for the resolution algo- rithm}

The algorithm computes an equilibrium solution. As explained in subsection 2.4, the computed equilibrium may be unstable and therefore unrealistic since any arbitrary small perturbation will lead to the modification of the response into one of the stable positions. Depending on both the surface and the adhesion potential, one or several equilibrium positions may exist and they may be either stable or unstable. The consequences on the algorithm are that the solution may strongly depend on the initialization and on the critical value for the stopping criterion. In other words, two different contact maps can be obtained from two different initializations. However, since this algorithm is giving an equilibrium solution, it prepares further stability analysis. This requires the computation of eigenvalues of the second derivative operator of the problem at the equilibrium position under the inequality contact constraints. This is the topic of ongoing work.

\section{Validation}

This section aims at validating the presented algorithm. We first compare the obtained numerical results with known analytical solutions for the Hertz problem without and with adhesion. Then, we illustrate the influence of adhesion on the contact clusters on a rough surface.

\subsection{Hertzian case}

The surface profile $h$ is given by:

$$
h(r)=-\frac{r^{2}}{2 R}
$$


and this surface is considered infinitely rigid. We study the elastic response of a flat surface of effective Young's modulus $E=1$. The radius $R$ of the indenter is equal to $2 L$ where $L$ stands for the length of the surface. In the presented calculations $L$ will be set to 1 . We will only consider small loads so that the periodic boundary conditions are not too influential. The stopping criterion is $\epsilon=\sum_{i=1}^{N} g_{i} q_{i}^{\prime}<10^{-9}$.

\subsubsection{Without adhesion}

Without adhesive forces, the exact axi-symmetric pressure and displacement solutions are given by:

$$
\left\{\begin{array}{l}
p_{e x}(r)=\frac{2 E}{\pi R} \sqrt{a^{2}-r^{2}} \\
u_{e x}(r)=h(r)+\frac{a}{\pi R} \sqrt{r^{2}-a^{2}}+\frac{r^{2}-2 a^{2}}{\pi R} \arccos \left(\frac{a}{r}\right)
\end{array}\right.
$$

where $a$ is the exact contact radius.

In Figure 1, we plot the profile at $y=0$ of displacement and pressure for the exact solution and the computed one for mean value of gap $g_{0}=0.0398 \mathrm{~L}$, which corresponds to the loading pressure $p_{0}=2 \cdot 10^{-4} \mathrm{E}$ in a $512 \times 512$ grid. Once again, we observe a good agreement between the two. The difference observed in displacements when $x \rightarrow L$ is due to the periodic boundary conditions (PBC) used in the simulation, while the Hertz model considers an infinite solid. by

In table 1 , we give the relative error at convergence, in energy norm $\eta$ defined

$$
\eta^{2}=\frac{\int_{S}\left(u-u_{e x}\right)\left(p-p_{e x}\right) d S}{\int_{S} u_{e x} p_{e x} d S}
$$

for 6 different discretizations.

Remark 1. Since $u$ is the approximate solution computed by collocation, $\eta$ is computed as following:

$$
\eta^{2}=\frac{\sum_{i=1}^{N}\left(u_{i}-u_{e x}\left(x_{i}\right)\right)\left(p_{i}-p_{e x}\left(x_{i}\right)\right)}{\int_{S} u_{e x} p_{e x} d S}
$$


We observe that the error for a $64 \times 64$ grid is already small and that refining the grid size reduces the error, although the reduction is not significant, once again because the numerical solution is slightly perturbed by the periodic boundary conditions.

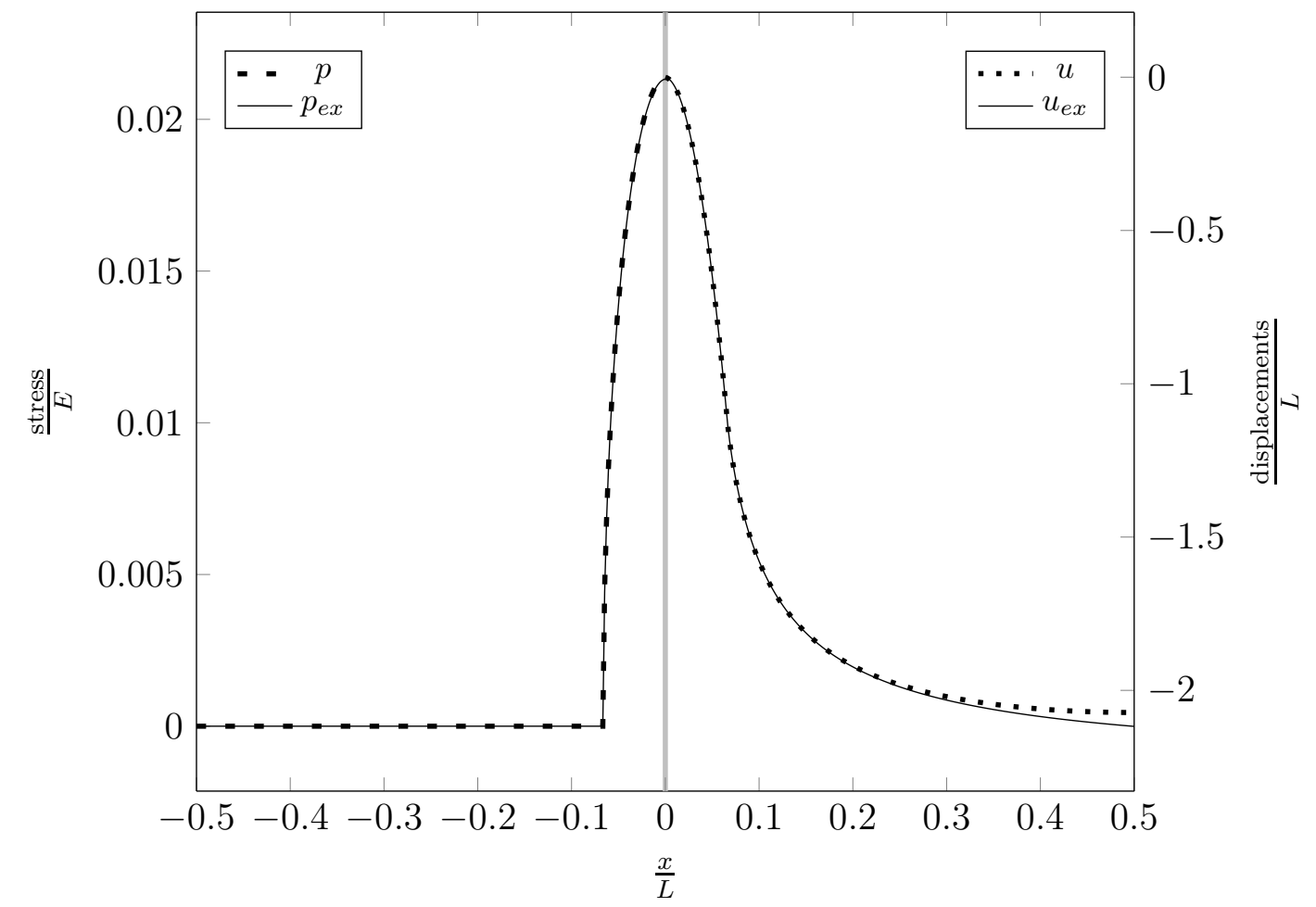

Figure 1: Comparison between analytical Hertzian solution and BEM computed solution on a $512 \times 512$ grid with load $p_{0}=2 \cdot 10^{-4} E$. On the left, pressure distributions are plotted and on the right displacements are plotted. The observed difference between displacements is due to the periodic boundary conditions in the BEM simulation.

We also implemented, in our home-made software, a standard penalization technique with the penalization parameter $k_{p e n}=5000$ and as well as an Uzawa algorithm. In addition to the relative error in energy norm, we give the relative error $\theta$ defined by:

$$
\theta^{2}=\frac{\int_{S}\left(u-u_{e x}\right)\left(u-u_{e x}\right) d S}{\int_{S} u_{e x} u_{e x} d S}
$$

Table 2 gathers the relative errors $\theta$ and $\eta$ and the minimum value of the 
gap at convergence for each method. We observe a negative gap for the penalty approach. The three methods give similar relative error in displacement but only the constrained gradient gives a small error in energy norm. This is due to the recovery of the mean value of the pressure at convergence. In Uzawa and penalty algorithms, since the orthogonality between gap and pressure is not ensured, wrong results are obtained while recovering the mean value $p_{0}$ using theorem 1 .

\subsubsection{Adhesive contact}

In order to compare with exact solutions for adhesive contact, we choose the simple Maugis-Dugdale adhesive potential [17] that considers that the adhesive force is constant if the gap between the two surfaces is smaller than the characteristic length $\rho$. Adhesion does not have any effect if the gap is larger than $\rho$. We note $\gamma$ the surface energy. As a consequence, the adhesive potential reads:

$$
E_{a d h}= \begin{cases}0 & \text { if } g>\rho \\ -\gamma \int_{S}\left(1-\frac{g(\underline{x})}{\rho} d S\right) & \text { if } g \leqslant \rho\end{cases}
$$

Therefore, the adhesive force derived from this potential is:

$$
f_{a d h}= \begin{cases}0 & \text { if } g>\rho \\ \frac{\gamma}{\rho} & \text { if } g \leqslant \rho\end{cases}
$$

This Maugis-Dugdale (MD) model also allows to study two asymptotic cases:

- the Johnson-Kendall-Roberts (JKR) approximation [15], which neglects adhesive forces outside the contact area and leads to an infinite elastic stress at the edge of contact, as in linear fracture mechanics. The JKR theory applies to large compliant spheres.

- the Derjaguin-Muller-Toporov (DMT) approximation [10], which assumes that adhesive forces can act outside the contact area but do not deform surfaces in the contact area. The DMT theory applies for small stiff spheres.

The Tabor coefficient $\mu_{T}$ is a dimensionless scalar parameter that enables to determine if the problem is close to the JKR approximation or to the DMT one. It is defined by:

$$
\mu_{T}=\frac{\gamma^{\frac{2}{3}} R^{\frac{1}{3}}}{\rho E^{\frac{2}{3}}}
$$

where $R$ is the typical local radius of curvature of the considered surface (here the radius of the sphere). Small $\mu_{T}$ corresponds to DMT approximation and large $\mu_{T}$ corresponds to JKR approximation. 
In the following, we will consider two cases for the adhesive parameters: ( $\rho=$ $\left.3.010^{-4} L, \gamma=1.010^{-5} E L\right)$ and $\left(\rho=2.010^{-3} L, \gamma=1.010^{-4} E L\right)$. The stiffness of the material is $E$ in both cases. The solution for the first set of parameters will be compared to the exact solution of the JKR model since the chosen values define a small Tabor coefficient and an effect of adhesion that exists only near the contact zone. The solution for the second set of parameters will be compared to the exact solution of the MD-model. The JKR solution is given by:

$$
\left\{\begin{array}{l}
p_{e x}(r)=\frac{2 E}{\pi R} \sqrt{a^{2}-r^{2}}-\frac{K_{I}}{\sqrt{\pi a}} \frac{a}{\sqrt{a^{2}-r^{2}}} \\
u_{e x}(r)=h(r)+\frac{a}{\pi R} \sqrt{r^{2}-a^{2}}+\frac{r^{2}-2 a^{2}}{\pi R} \arccos \left(\frac{a}{r}\right)+\frac{2 K_{I}}{\pi E} \sqrt{\pi a} \arccos \left(\frac{a}{r}\right)
\end{array}\right.
$$

where $K_{I}=\sqrt{\frac{2 \gamma}{E}}$. One clearly sees the stress singularity at $r=a$. Of course, the infinite value of the stress at $r=a$ cannot be captured by numerical simulation.

In table 3, we give the relative error in energy norm for 5 different discretizations. Contrary to the non-adhesive case, the $64 \times 64$ surface does not give a good approximation of the solution. It illustrates that adhesion is more demanding in terms of discretization: the grid has to represent correctly the new parameter $\rho$ which can be quite small.

In figure 2, we give the profile of both pressure and displacement for computed and exact solutions on a $2048 \times 2048$ grid. The agreement between the two is very good and the difference is due to the non-zero length $\rho$ in the simulation, the discretization and the periodic boundary conditions.

The MD exact solution is given by:

$$
\left\{\begin{aligned}
p_{e x}(r) & =\frac{2 E}{\pi R} \sqrt{a^{2}-r^{2}}-\frac{2 \sigma_{0}}{\pi} \arctan \left(\sqrt{\frac{c^{2}-a^{2}}{a^{2}-r^{2}}}\right) \\
u_{e x}(r) & =h(r)+\frac{a}{\pi R} \sqrt{r^{2}-a^{2}}+\frac{r^{2}-2 a^{2}}{\pi R} \arccos \left(\frac{a}{r}\right) \\
& +\frac{4 \sigma_{0}}{E \pi a}\left[a c^{2} \int_{a}^{\min (r, c)} \frac{\sqrt{r^{2}-t^{2}}}{t^{2} \sqrt{c^{2}-t^{2}}} d t-\sqrt{c^{2}-a^{2}}\left(\sqrt{r^{2}-a^{2}}-a \arccos \left(\frac{a}{r}\right)\right)\right]
\end{aligned}\right.
$$

To obtain the relationship between the model parameters, the load, the contact area and the adhesive zone radius $c$, it is necessary to solve iteratively a non-linear equation that connects all these scalars. To avoid this difficulty, we chose to use the quasi-analytical formula proposed in [8]. 


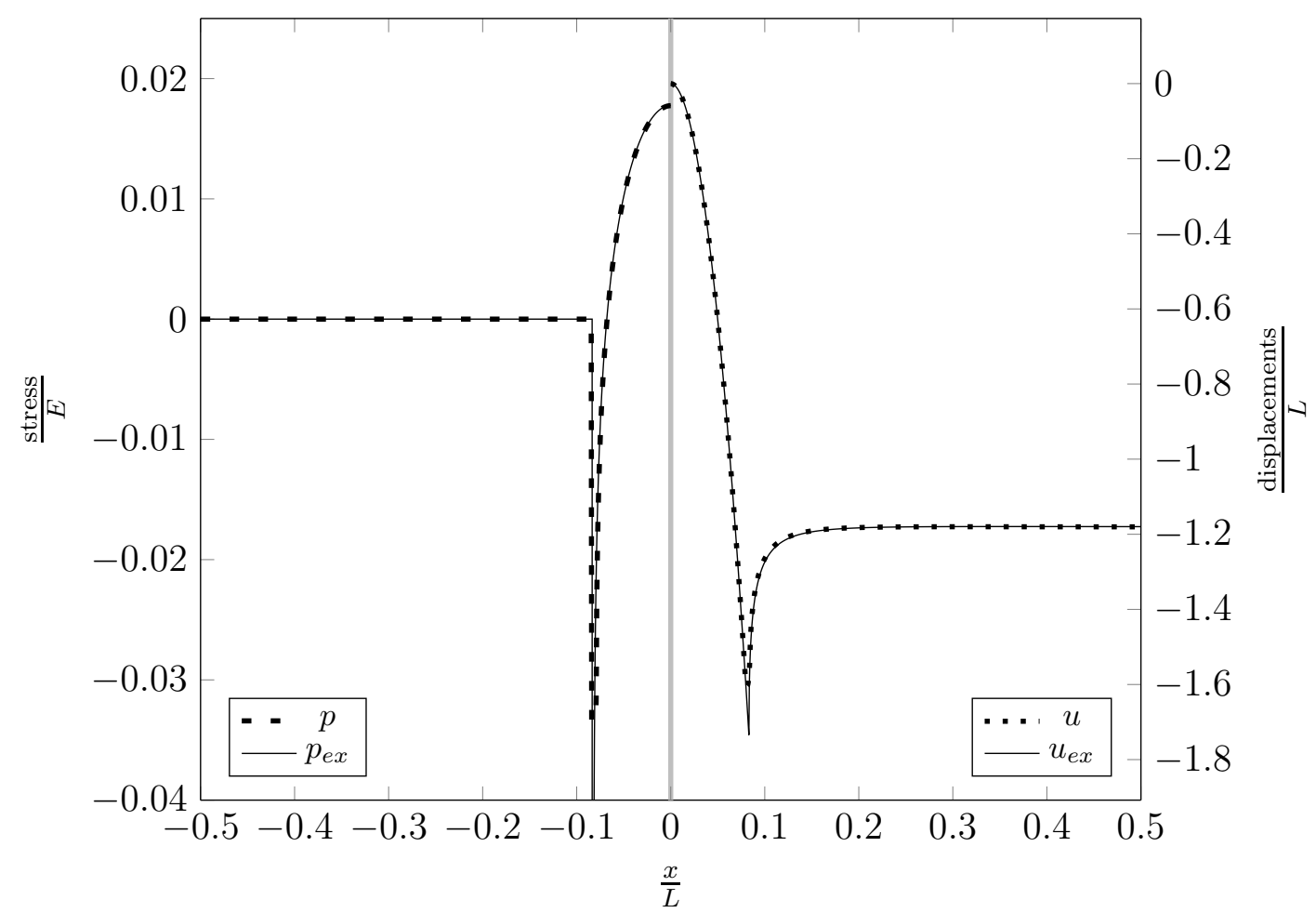

Figure 2: Comparison between analytical solution of the JKR adhesive model on Hertzian contact and BEM computed solution for a MD adhesive potential with characteristic length for adhesion $\rho=3 \cdot 10^{-4} L$ on a $2048 \times 2048$ grid with mean value of gap $g_{0}=4.0510^{-2} \mathrm{~L}$. The difference observed is due to the non-zero length $\rho$ in the simulation, the discretization and the PBC.

In table 4 , we give the relative error in energy norm for 5 different discretizations. Once again, we observe that adhesion is more demanding in terms of discretization.

In figure 3 we give the profile of both pressure and displacement for computed and exact solutions on a $1024 \times 1024$ grid. We observe that the computed solution is very close to the exact solution. The zone with negative pressure is larger than in the JKR case, which is consistent with a larger value of $\rho$.

Finally, we give on the same Figure 4, the estimation of convergence rates for the three presented cases.

\subsection{Adhesive contact between rough surfaces}

In this section we compute the solution of the adhesive contact on a rough surface for non-adhesive contact (zero Tabor coefficient) and adhesive contact. 


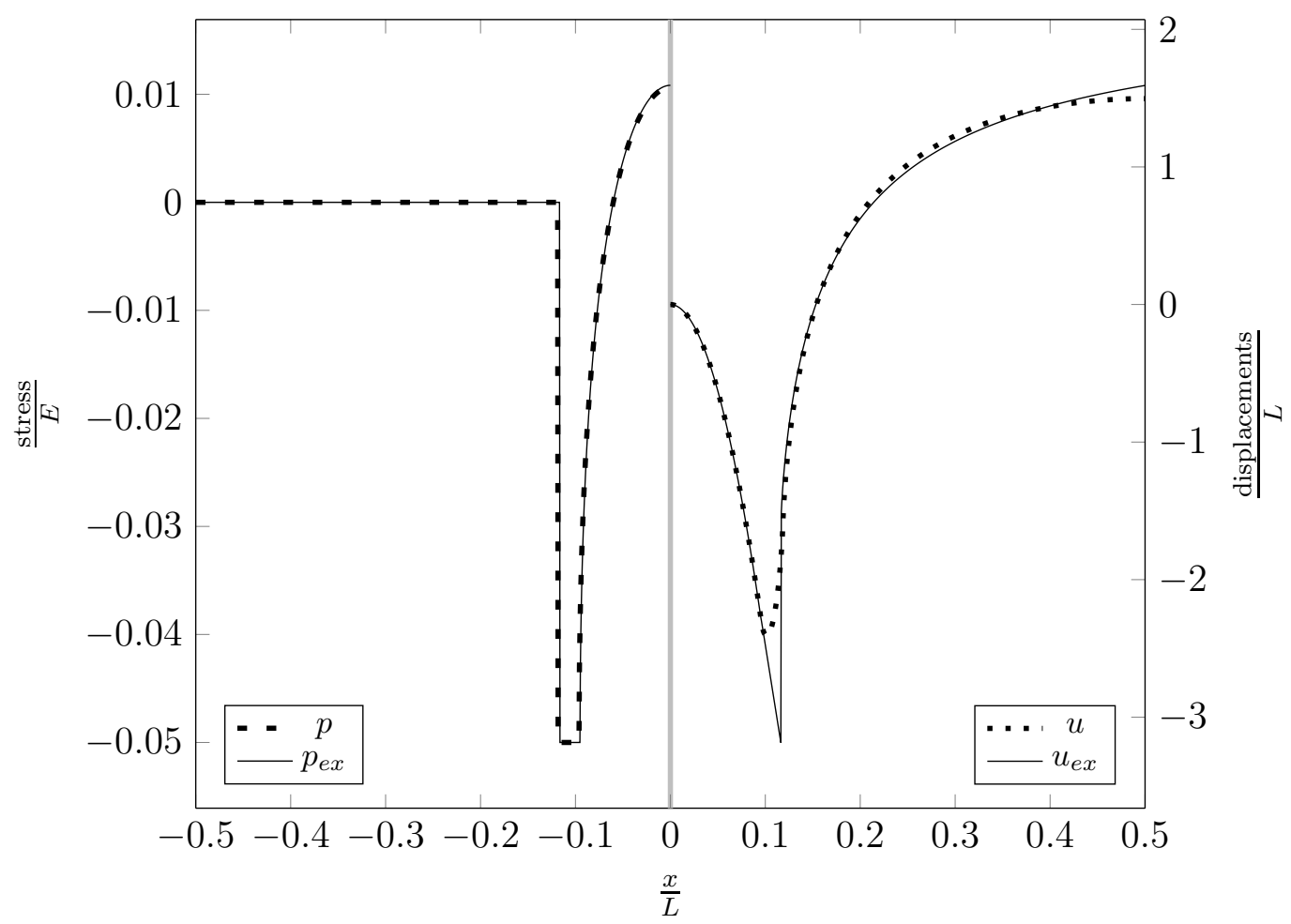

Figure 3: Comparison between analytical solution of the MD adhesive model on Hertzian contact and BEM computed solution for a MD adhesive potential with characteristic length for adhesion $\rho=2 \cdot 10^{-3} L$ on a $2048 \times 2048$ grid with mean value of gap $g_{0}=4.2910^{-2} \mathrm{~L}$. The difference observed is due to the discretization and the PBC.

First we describe the rough surface and the way we generate it. Then we give the contact maps obtained in presence and absence of adhesion.

\subsubsection{Description of the rough surface}

A rough surface is usually described by its auto-correlation function $R_{h}(\underline{x})$

$$
R_{h}(\underline{x})=\frac{1}{L^{2}} \int_{S} h\left(\underline{x}-\underline{x}^{\prime}\right) h\left(\underline{x}^{\prime}\right) d \underline{x}^{\prime}
$$

or by its power spectral density (PSD), which is the Fourier transform of the auto-correlation function [16].

To define the form of the PSD for a rough surface, we introduce the following wavelengths: $\lambda_{s}$ (shortest wavelength), $\lambda_{r}$ (roll-off wavelength) and $\lambda_{l}$ (largest 


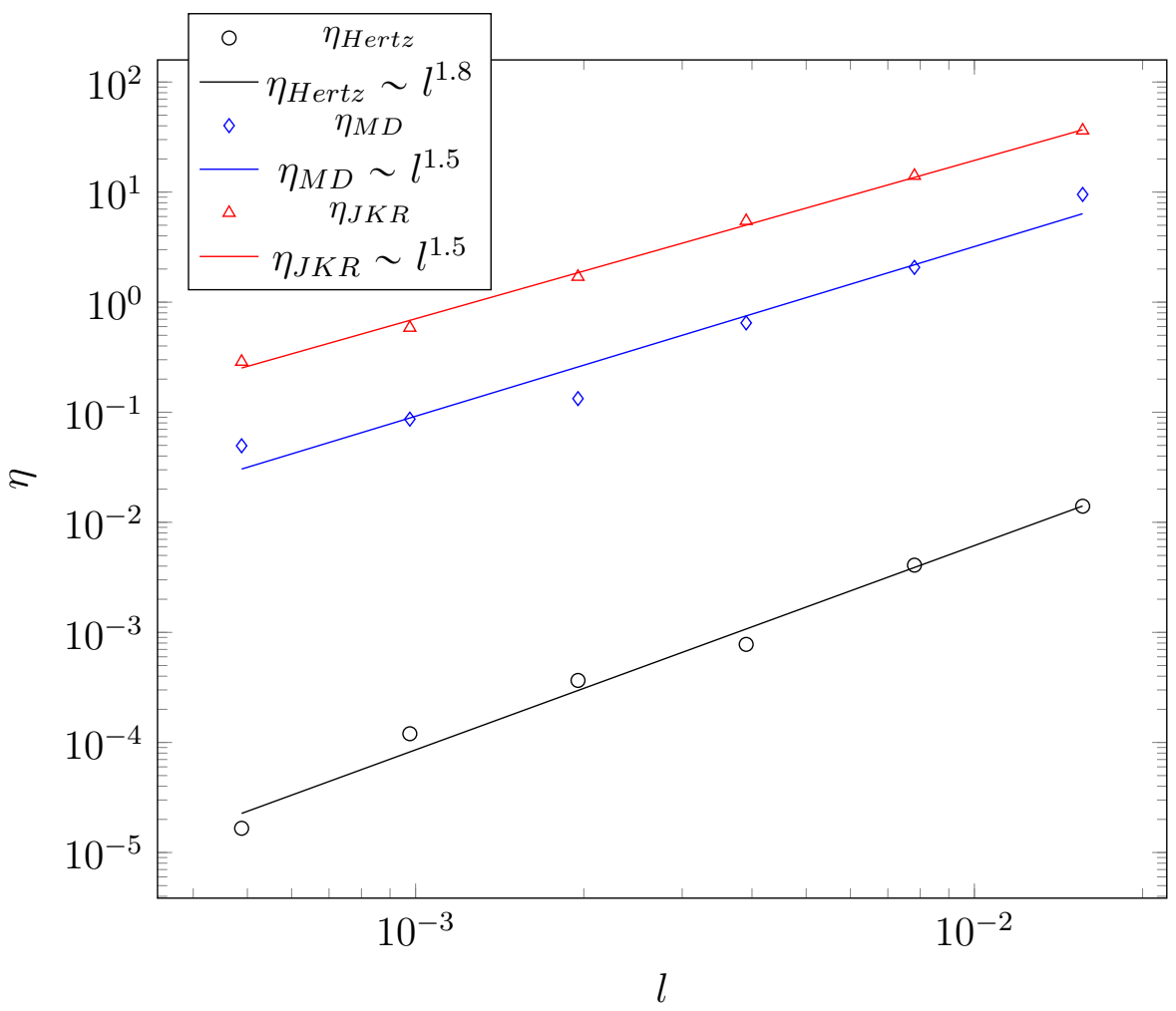

Figure 4: Errors and estimation of convergence rates for Hertz problem without adhesion $\left(\eta_{\text {Hert } z}\right)$ and with adhesion $\left(\eta_{M D}, \eta_{J K R}\right)$ : including adhesion lower the convergence rate : the exponent goes from 1.8 to 1.5 .

wavelength). Therefore, we also have the following wave numbers: $k_{s}=\frac{L}{\lambda_{s}}, k_{r}=\frac{L}{\lambda_{r}}$ and $k_{l}=\frac{L}{\lambda_{l}}$.

The power spectral density of natural rough surfaces often follows a self-affine behavior:

$$
\Phi(|\mathbf{k}|)= \begin{cases}C & \text { if } k_{l} \leqslant|\mathbf{k}| \leqslant k_{r} \\ C\left(\frac{|\mathbf{k}|}{k_{r}}\right)^{-2-H} & \text { if } k_{l} \leqslant|\mathbf{k}| \leqslant k_{r} \\ 0 & \text { otherwise }\end{cases}
$$

where $\mathrm{H}$ is the Hurst exponent and $\mathrm{C}$ the roughness amplitude. $|\mathbf{k}|$ is the norm of the wave vector:

$$
|\mathbf{k}|=\sqrt{k_{x}^{2}+k_{y}^{2}}
$$

To construct periodic rough surfaces, we use a Fourier-based filtering algorithm [13], which can be summarized as follows: 
1. Generate random heights $\eta(x, y)$ according to an isotropic normal law (Gaussian white noise)

2. Compute the PSD $S\left(k_{x}, k_{y}\right)=S$ of $\eta$ (should be constant)

3. Define the PSD $\Phi\left(k_{x}, k_{y}\right)$ of the desired surface from the parameters

4. Compute

$$
H\left(k_{x}, k_{y}\right)=\frac{\Phi\left(k_{x}, k_{y}\right)}{S}
$$

5. Compute the product

$$
Z=\left(H\left(k_{x}, k_{y}\right)\right) \operatorname{FFT}(\eta)
$$

6. Compute the backward Fourier transform

$$
h(x, y)=\mathrm{FFT}^{-1}(Z)
$$

We used this filtering process to generate one rough surface whose properties are: $L=1, k_{l}=4, k_{r}=4, k_{s}=64, H=0.8$ and the root mean square of slopes is 0.001 . The surface size is $1024 \times 1024$. The choice of $k_{l}$ and $k_{r}$ different from 1 is justified by the need to have a representative surface (see [31] for a detailed discussion on this point). The spectrum of the surface is not as rich as the one of truly rough surfaces. However, adhesion is very demanding in terms of discretization. Since we do not aim at a comprehensive parametric study on the influence of wave numbers on adhesive contact in this article, we chose a smaller spectrum but with a sufficient discretization $(1024 \times 1024)$. In figure 5 , we give a representation of the rough surface.

\subsubsection{Contact maps}

We consider the following adhesion energy:

$$
E_{a d}=-\gamma \int_{S} \exp \left(\frac{-g}{\rho}\right) d S
$$

where $\gamma$ is the surface energy and $\rho$ the characteristic length of range of adhesive effect. The adhesive force is therefore:

$$
f_{a d h}(\underline{x})=\frac{\partial E_{a d}}{\partial u}=\frac{\gamma}{\rho} \exp \left(\frac{-g(\underline{x})}{\rho}\right)
$$




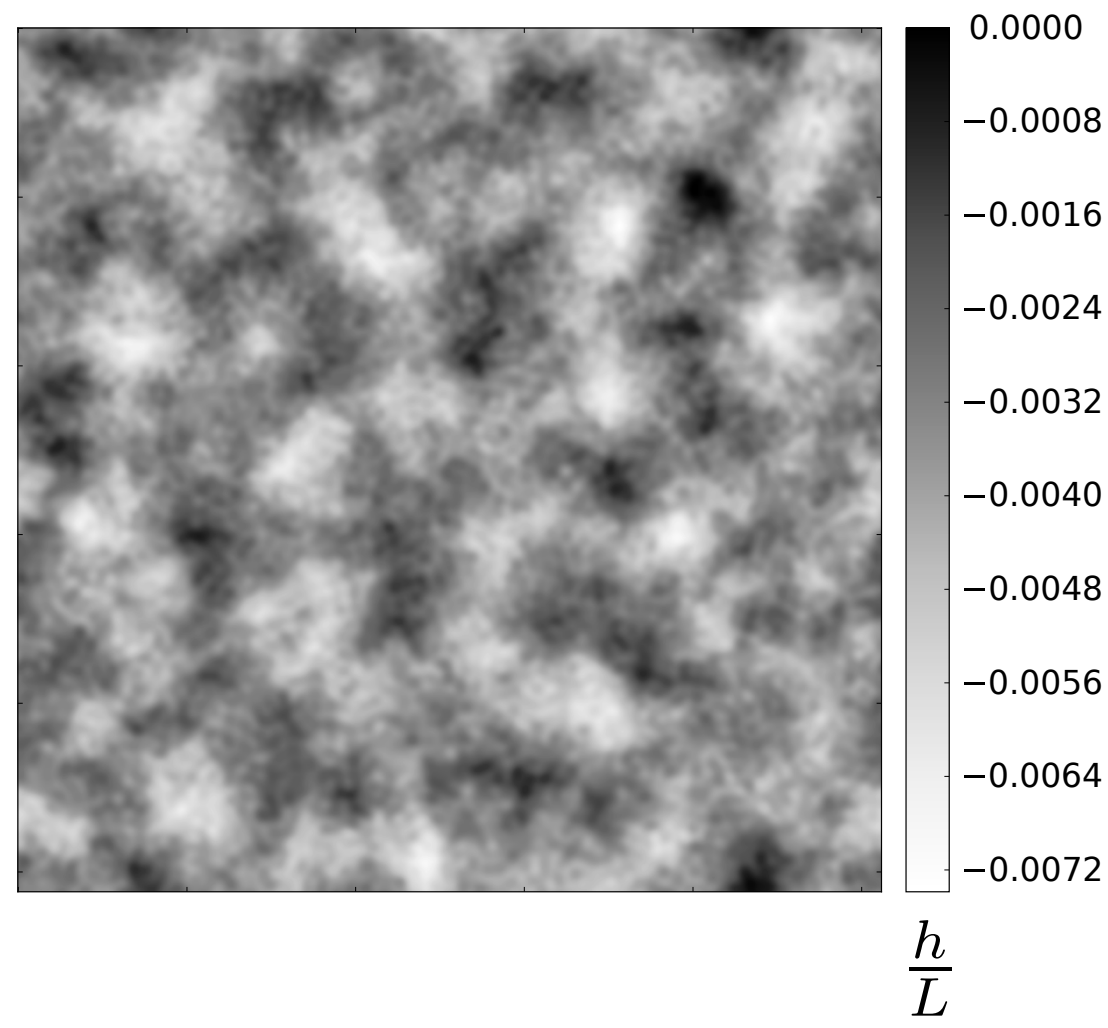

Figure 5: Heights of the rough surface

This potential has been studied in [18] with a GFMD [2] method. Adhesion is proportional to surface energy and the closer the surfaces, the larger the adhesion effect. Moreover, with this model, the adhesion force can be very small but never null.

In the first computation we set $\gamma=0$ and there will be no adhesion. For the second computation, we chose $\gamma=2.010^{-5} E L^{3}$ and $\rho=2.010^{-5} \mathrm{~L}$.

In Figure 6, we give the signed pressure maps obtained on the same surface for the same mean load $q_{0}=0.003 E$. On the first map, we observe only zero pressure (in grey) and positive pressure (in black) since no adhesion is considered. On the second map, we clearly see that the zone of negative pressure (in white) surrounds the positive pressure clusters. In Figure 7, we give the associated contact maps (contact is in black). One clearly sees that in presence of adhesion, the contact area is bigger than without adhesion for the same mean value of pressure $q_{0}$.

Uzawa and penalty algorithms diverge and cannot compute solution for this problem. This can be explained by the exponential adhesive potential, which strongly changes the functional to minimize even for a slightly negative gap. Moreover, the rough surface is a much more complicated geometry than the single 

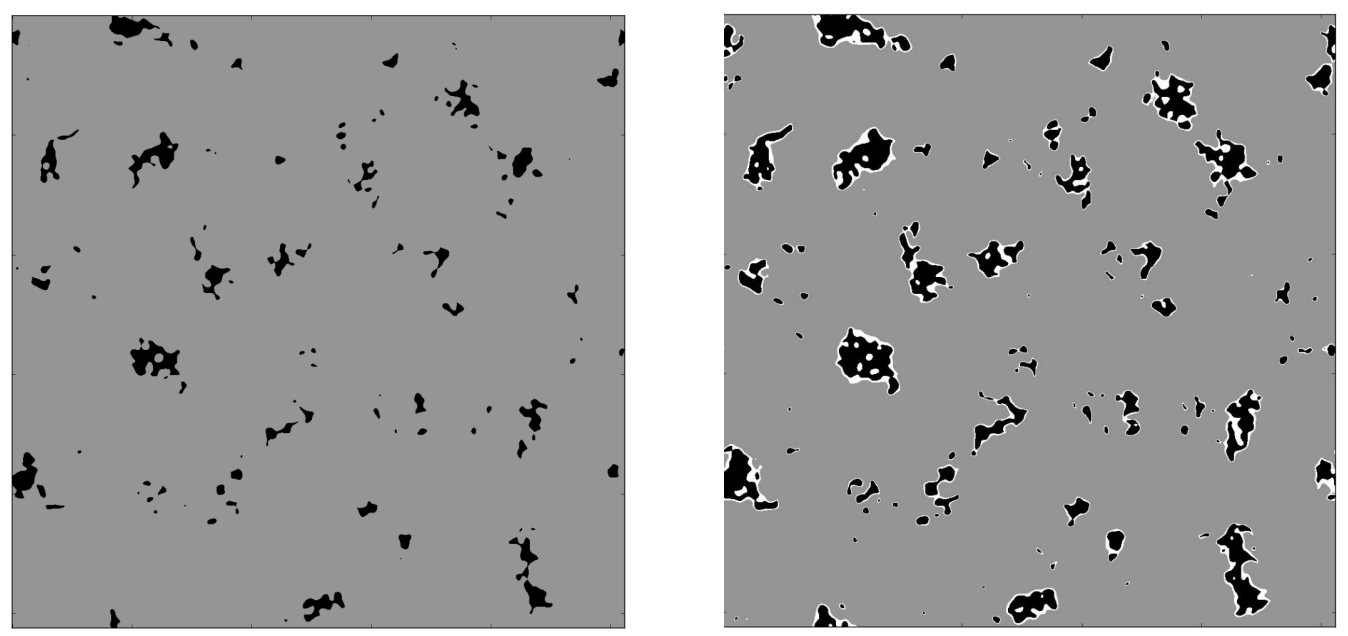

Figure 6: Pressures without and with adhesion. Grey is 0 pressure, black is positive pressure and white is negative pressure

asperity case of Hertz contact.

Finally, with the hypothesis that stability issues have no influence on the global true contact area, we give the repulsive contact area as a function of the load for different surface energy $\gamma$ in Figure 8. As expected, in absence of adhesion $(\gamma=0)$, the contact area depends linearly on the ratio $\frac{q_{0}}{E h_{r m s}^{\prime} A_{0}}$, where $A_{0}=L^{2}$ is the nominal area, where $q_{0}$ is the mean value of the sum of adhesive and elastic pressures and where $h_{r m s}^{\prime}$ is the root mean square of the slopes of the surface. Adhesion increases the contact area, which results in larger slope $\kappa$ (estimated using the origin tangent). This trend was also observed in [21].

\section{Conclusion}

In this paper, we proposed an efficient algorithm to compute the solution of an adhesive normal contact problem solved with BEM on arbitrary rough surfaces. This algorithm ensures the contact orthogonality and is easy to implement. We validated it on the adhesive Hertzian case: the numerical results are in good agreement with analytical solutions. Finally, we illustrate the algorithm's robustness by solving the adhesive normal contact between one flat solid and a rough surface with an exponential adhesive law.

The main limitation of this method is that the two surfaces cannot be deformable simultaneously. Future work will consist in studying the stability of the equilibrium solution and the consequences on the geometry of the contact map. 

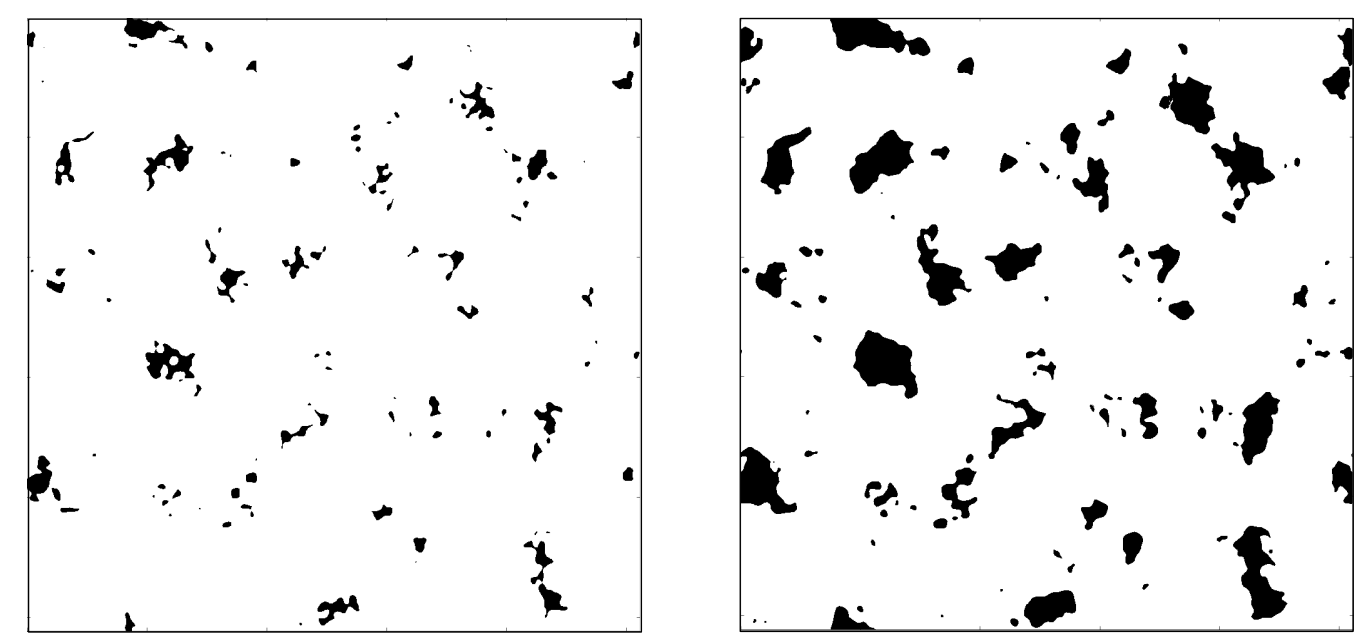

Figure 7: Contact area without and with adhesion. Contact zone (in black) is larger in case of adhesion than without.

\section{Acknowledgments}

Support for V.R. from the EPFL Fellows fellowship programme co-funded by Marie Skodowska-Curie, Horizon 2020 Grant agreement no. 665667 is gratefully acknowledged.

\section{References}

[1] Bemporad, A., Paggi, M.: Optimization algorithms for the solution of the frictionless normal contact between rough surfaces. International Journal of Solids and Structures 69, 94-105 (2015)

[2] Campañá, C., Müser, M.H.: Practical green's function approach to the simulation of elastic semi-infinite solids. Physical Review B 74, 075,420 (2006)

[3] Campañá, C., Müser, M.H., Robbins, M.O.: Elastic contact between selfaffine surfaces: comparison of numerical stress and contact correlation functions with analytic predictions. Journal of Physics-Condensed Matter 20(35), $354,013+(2008)$

[4] Carbone, G., Bottiglione, F.: Asperity contact theories: Do they predict linearity between contact area and load? J Mech Phys Solids 56(8), 25552572 (2008) 


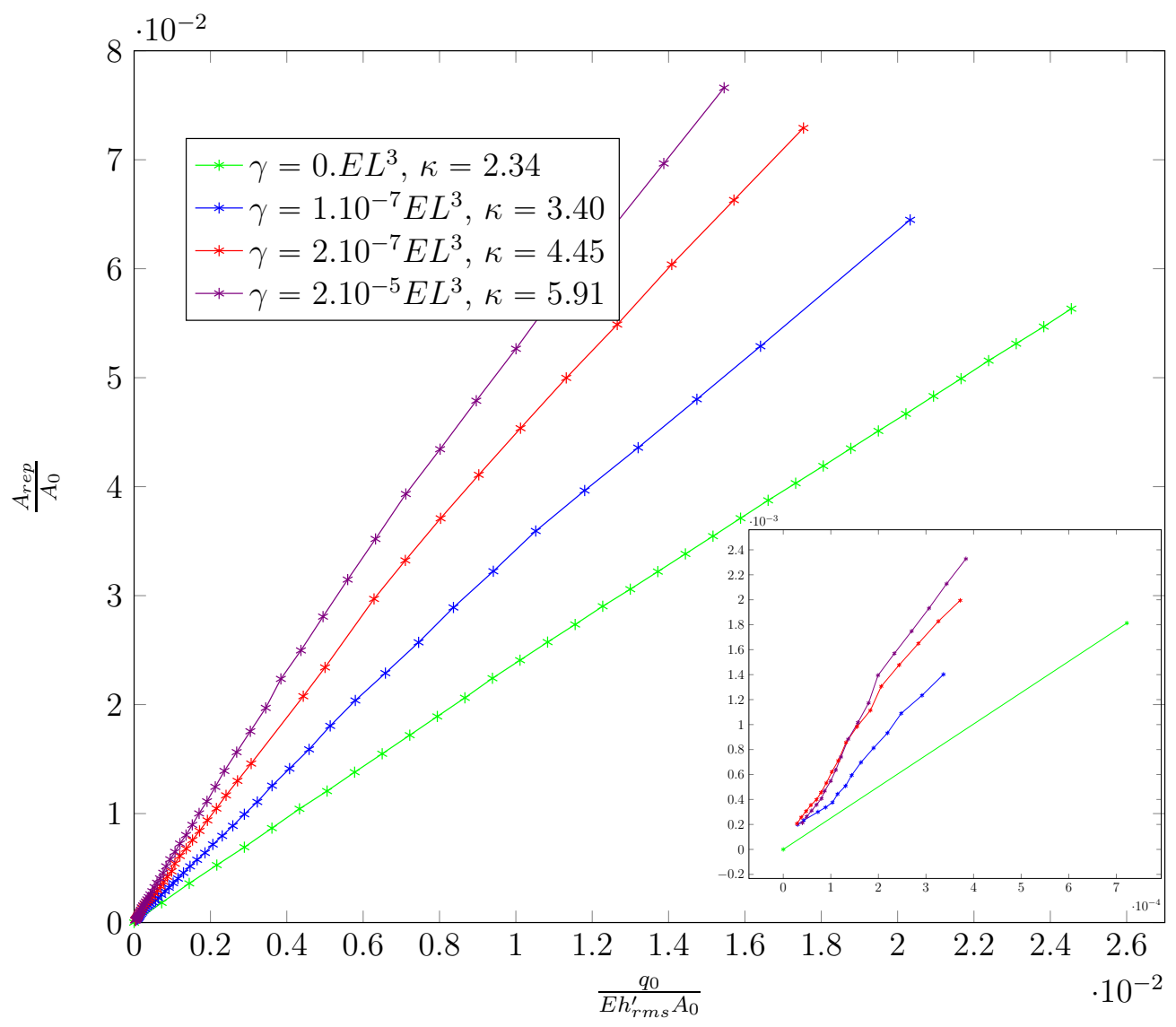

Figure 8: Repulsive contact area as a function of load for different surface energy $\gamma$. The slope $\kappa$ is given in the legend. On the bottom right-hand corner, a zoom on the origin shows that only the non-adhesive curve intersects $(0,0)$ 
[5] Carbone, G., Mangialardi, L.: Adhesion and friction of an elastic half-space in contact with a slightly wavy rigid surface. Journal of the Mechanics and Physics of Solids 52(6), 1267 - 1287 (2004)

[6] Carbone, G., Mangialardi, L.: Analysis of the adhesive contact of confined layers by using a green's function approach. Journal of the Mechanics and Physics of Solids 56(2), $684-706$ (2008)

[7] Carbone, G., Scaraggi, M., Tartaglino, U.: Adhesive contact of rough surfaces: Comparison between numerical calculations and analytical theories. European Physical Journal E 30, 65-74 (2009)

[8] Carpick, R.W., Ogletree, D.F., Salmeron, M.: A general equation for fitting contact area and friction versus load measurements. Journal of Colloid and Interface Science 211, 395-400 (1978)

[9] Chaudhury, M.K.: Interfacial interaction between low-energy surfaces. Materials Science and Engineering: R: Reports 16(3), 97-159 (1996)

[10] Derjaguin, B., Muller, V., Toporov, Y.: Effect of contact deformations on the adhesion of particles. Journal of Colloid and Interface Science 53(2), $314-$ $326(1975)$

[11] Greenwood, J.A.: A simplified elliptic model of rough surface contact. Wear 261(2), 191-200 (2006)

[12] Greenwood, J.A., Williamson, J.B.P.: Contact of nominally flat surfaces. Proceedings of the Royal Society of London A 295, 300-319 (1966)

[13] Hu, Y.Z., Tonder, K.: Simulation of 3-D random rough surface by 2-D digital filter and fourier analysis. International Journal of Machine Tools and Manufacture 32, 83-90 (1992)

[14] Hyun, S., Pei, L., Molinari, J.F., Robbins, M.O.: Finite-element analysis of contact between elastic self-affine surfaces. Physical Review E 70(2), 026,117+ (2004)

[15] K. L. Johnson, K.K., Roberts, A.D.: Surface energy and the contact of elastic solids. Proceedings of the Royal Society of London A 324(1558), 301-313 (1971)

[16] Longuet-Higgins, M.S.: Statistical properties of an isotropic random surface. Philosophical Transactions of the Royal Society A 250(975), 157-174 (1957) 
[17] Maugis, D.: Adhesion of spheres: The JKR-DMT transition using a Dugdale model. Journal of Colloid and Interface Science 150(1), 243-269 (1992)

[18] Müser, M.H.: Single-asperity contact mechanics with positive and negative work of adhesion: Influence of finite-range interactions and a continuum description for the squeeze-out of wetting fluids. Beilstein Journal of Nanotechnology 5, 419-437 (2014)

[19] Nguyen, Q.S.: Stability and nonlinear solid mechanics (2000)

[20] Paggi, M., Ciavarella, M.: The coefficient of proportionality k between real contact area and load, with new asperity models. Wear 268(7-8), 1020-1029 (2010)

[21] Pastewka, L., Robbins, M.O.: Contact between rough surfaces and a criterion for macroscopic adhesion. Proceedings of the National Academy of Sciences 111(9), 3298-3303 (2014)

[22] Pei, L., Hyun, S., Molinari, J.F., Robbins, M.O.: Finite element modeling of elasto-plastic contact between rough surfaces. Journal of the Mechanics and Physics of Solids 53(11), 2385-2409 (2005)

[23] Persson, B.N.J.: Theory of rubber friction and contact mechanics. Journal of Chemical Physics 115(8), 3840-3861 (2001)

[24] Persson, B.N.J.: Adhesion between elastic bodies with randomly rough surfaces. Physical Review Letters 89, 245,502 (2002)

[25] Polonsky, I., Keer, L.: A numerical method for solving rough contact problems based on the multi-level multi-summation and conjugate gradient techniques. Wear 231(2), 206 - 219 (1999)

[26] Putignano, C., Afferrante, L., Carbone, G., Demelio, G.: A new efficient numerical method for contact mechanics of rough surfaces. International Journal of Solids and Structures 42, 338-343 (2012)

[27] Signorini, A.: Sopra alcune questioni di elastostatica. Atti della Societa Italiana per il Progresso delle Scienze (1933)

[28] Stanley, H.M., Kato, T.: An FFT-based method for rough surface contact. Journal of Tribology 119, 481-485 (1997)

[29] Westergaard, H.: Bearing pressures and cracks. Journal of Applied Mechanics 6, 49-53 (1937) 
[30] Wriggers, P.: Computational contact mechanics. Springer Science \& Business Media (2006)

[31] Yastrebov, V.A., Anciaux, G., Molinari, J.F.: From infinitesimal to full contact between rough surfaces: Evolution of the contact area. International Journal of Solids and Structures 52, 83 - 102 (2015) 


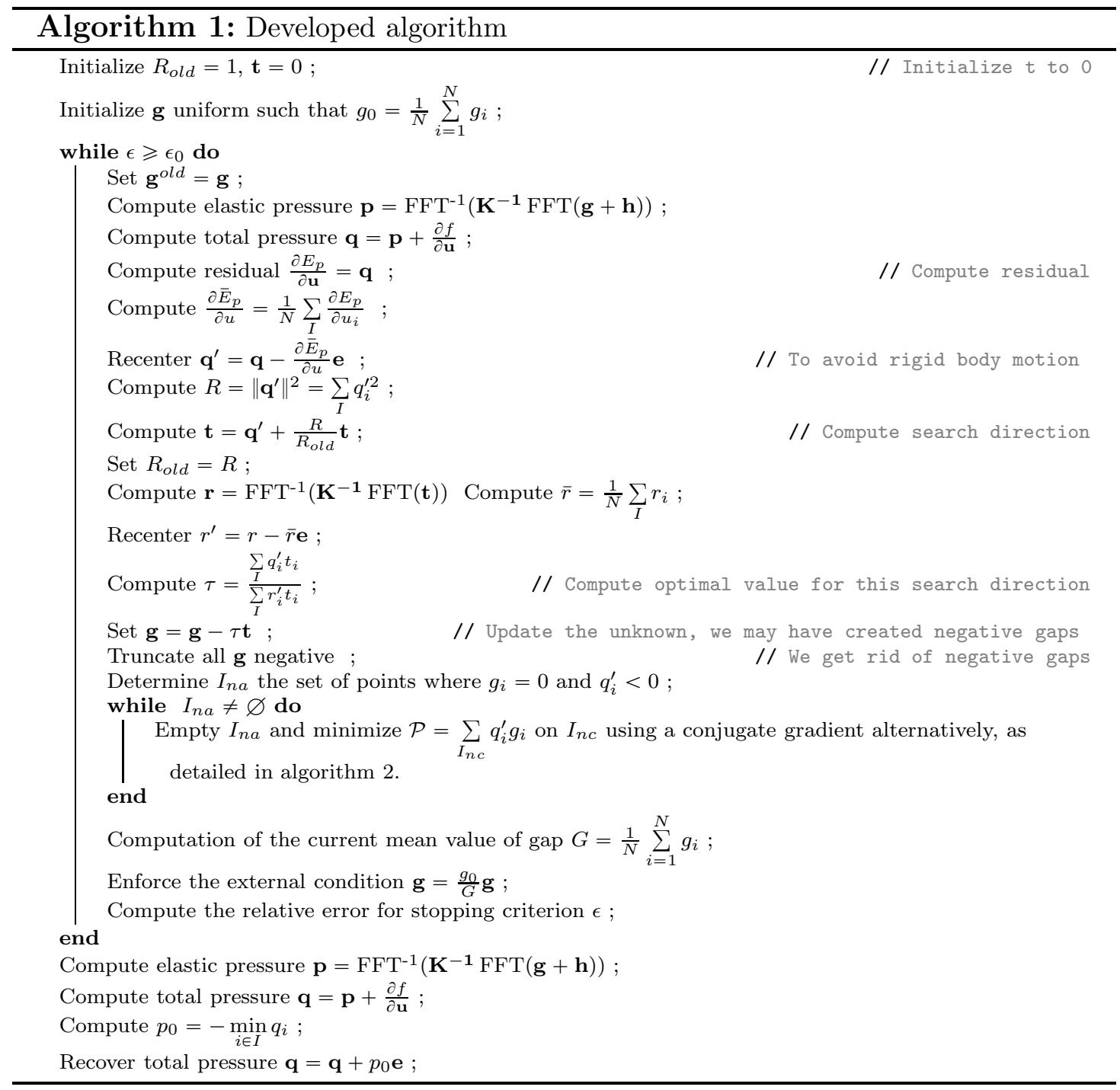




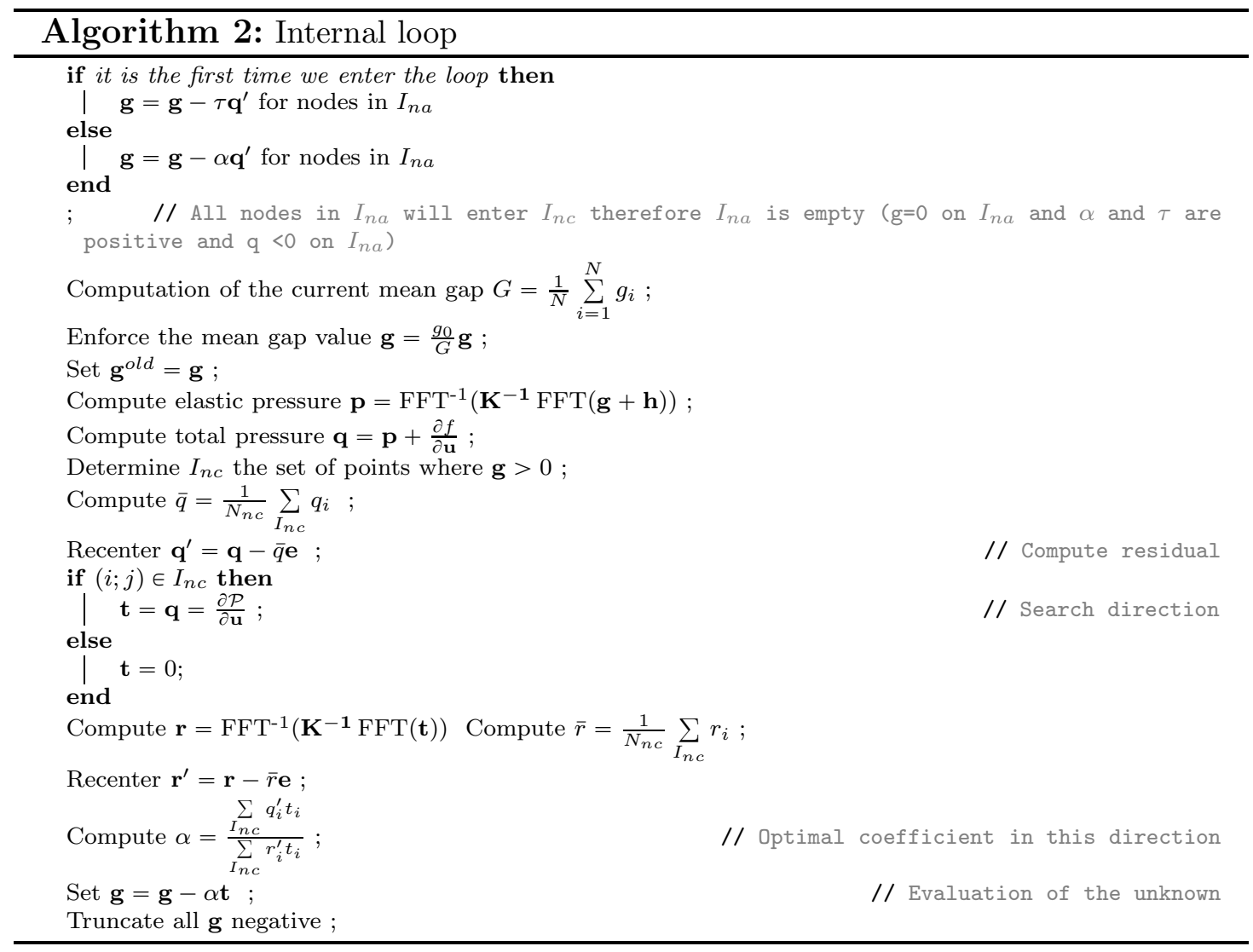




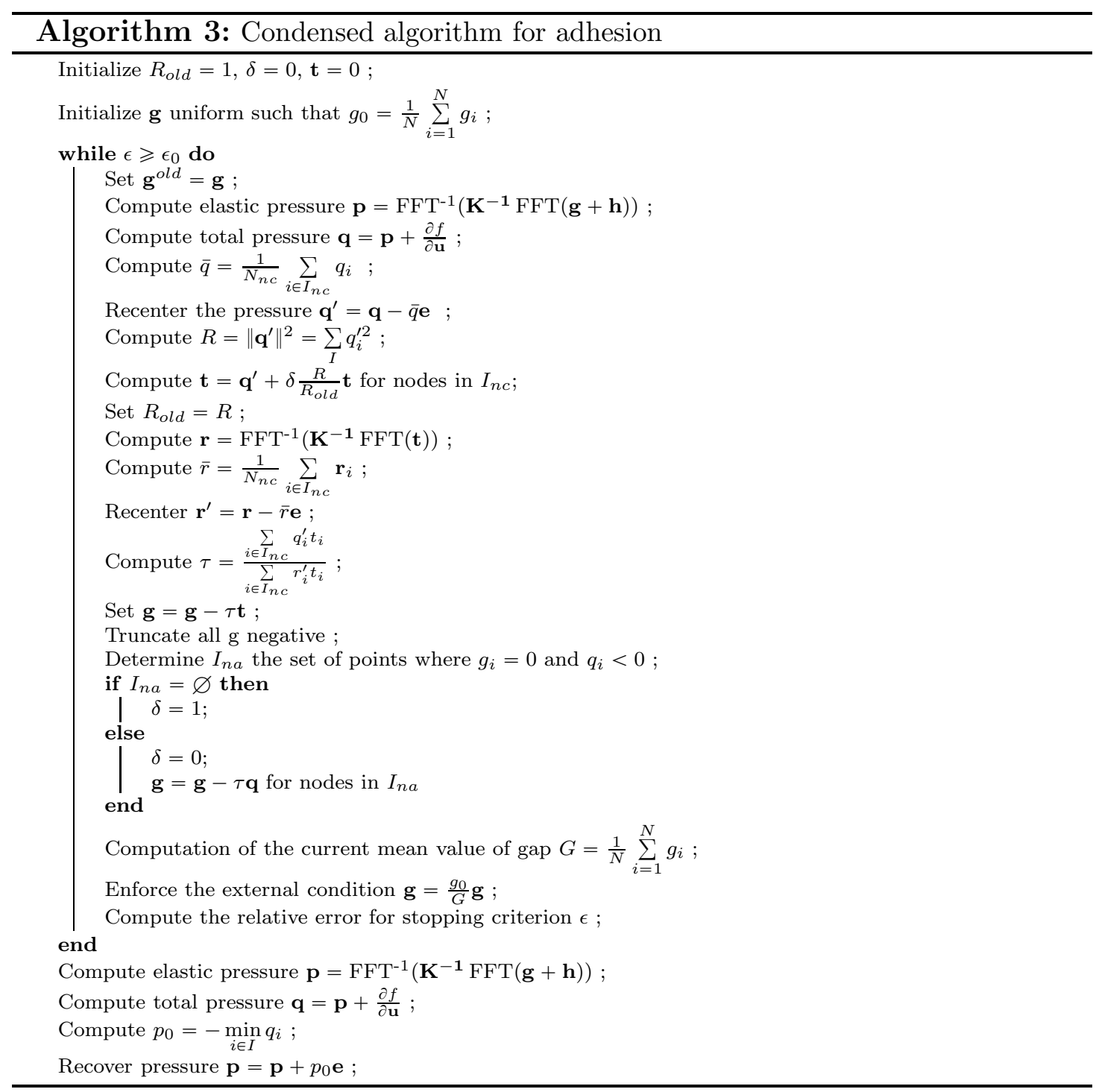

\begin{tabular}{|c|c|c|c|c|c|c|}
\hline grid size & $64 \times 64$ & $128 \times 128$ & $256 \times 256$ & $512 \times 512$ & $1024 \times 1024$ & $2048 \times 2048$ \\
\hline$\eta_{\text {Hertz }}$ & $1.4010^{-2}$ & $4.0810^{-3}$ & $7.7810^{-4}$ & $3.6610^{-4}$ & $1.2010^{-4}$ & $1.6610^{-5}$ \\
\hline
\end{tabular}

Table 1: Error in energy norm between computed and exact Hertz solution for different grid sizes: $64 \times 64$ grid already gives satisfying results. The numerical solution tends to the analytical solution when $l$ tends to 0 . 


\begin{tabular}{|c|c|c|c|}
\hline method & $\eta$ & $\theta$ & $\min (\mathbf{g})$ \\
\hline Penalty & 10 & $210^{-4}$ & $-410^{-6}$ \\
\hline Uzawa & 54 & $1.910^{-4}$ & machine precision \\
\hline Constrained gradient & $1.310^{-7}$ & $1.510^{-4}$ & machine precision \\
\hline
\end{tabular}

Table 2: Different relative error at the end of three algorithms: the constrained gradient approach gives the best results.

\begin{tabular}{|c|c|c|c|c|c|c|}
\hline grid size & $64 \times 64$ & $128 \times 128$ & $256 \times 256$ & $512 \times 512$ & $1024 \times 1024$ & $2048 \times 2048$ \\
\hline$\eta_{J K R}$ & 36.35 & 14.06 & 5.47 & 1.70 & 0.5856 & 0.2876 \\
\hline
\end{tabular}

Table 3: Error in energy norm between computed and exact JKR solution for different grid sizes: Adhesive contact is more demanding in dicretization than non-adhesive one.

\begin{tabular}{|c|c|c|c|c|c|c|}
\hline grid size & $64 \times 64$ & $128 \times 128$ & $256 \times 256$ & $512 \times 512$ & $1024 \times 1024$ & $2048 \times 2048$ \\
\hline$\eta_{M D}$ & 9.52 & 2.07 & $6.4810^{-1}$ & $1.3310^{-1}$ & $8.6510^{-2}$ & $4.9510^{-2}$ \\
\hline
\end{tabular}

Table 4: Error in energy norm between computed and exact MD solution for different grid sizes: Adhesive contact is more demanding in dicretization than non-adhesive one. 